\title{
METTL14 promotes the migration and invasion of breast cancer cells by modulating N6-methyladenosine and hsa-miR-146a-5p expression
}

\author{
DANDAN YI $^{1 *}$, RU WANG $^{2 *}$, XIANBIAO SHI $^{1}$, LEI XU ${ }^{2}$, YIMINU'ER YILIHAMU $^{3}$ and JIANFENG SANG ${ }^{1}$ \\ ${ }^{1}$ Department of General Surgery, The Affiliated Drum Tower Hospital of Nanjing University Medical School, Nanjing, \\ Jiangsu 210008; ${ }^{2}$ Department of General Surgery, Drum Tower Clinical Medical College of Nanjing Medical University, \\ Nanjing, Jiangsu 211166; ${ }^{3}$ Department of General Surgery, Drum Tower Clinical Medical College of Nanjing University, \\ Nanjing, Jiangsu 210093, P.R. China
}

Received June 14, 2019; Accepted January 7, 2020

DOI: $10.3892 / o r .2020 .7515$

\begin{abstract}
Breast cancer (BC) is the most frequently diagnosed cancer and the leading cause of cancer-related death among women worldwide. Evidence indicates that posttranscriptional N6-methyladenosine (m6A) modification modulates BC development. In the present study, we assessed BC and normal tissues to investigate this connection. RNA m6A levels were determined by methylation quantification assay. The effects of methyltransferase-like 14 (METTL14) gain-of-expression or co-transfection with an m6A inhibitor on cell migration and invasion abilities were determined by Transwell assays. The levels of differentially expressed (DE) miRNAs were verified by real-time quantitative PCR (RT-qPCR). Gene Ontology (GO) and Kyoto Encyclopedia of Genes and Genomes analyses (KEGG) were performed to analyze potential function of target genes of the DE miRNAs. The effects of candidate miRNAs modulated by METTL14 on cell migration and invasion abilities were confirmed by Transwell assays. We demonstrated that m6A methyltransferase METTL14 was significantly upregulated in BC tissues compared with normal tissues. METTL14 gain- and loss-of-expression regulated m6A levels in MCF-7 and MDA-MB-231 cells. The cell function assays revealed that METTL14 overexpression enhanced the migration and invasion capacities of BC cells. Moreover, treatment with the m6A inhibitor suppressed this enhanced cell migration and invasion. Additionally, aberrant expression of METTL14 reshaped the miRNA profile in BC cell lines. The remodeled DE miRNA/mRNA network was found
\end{abstract}

Correspondence to: Dr Jianfeng Sang, Department of General Surgery, The Affiliated Drum Tower Hospital of Nanjing University Medical School, 321 Zhongshan Road, Gulou, Nanjing, Jiangsu 210008, P.R. China

E-mail: drsangjianfeng@163.com

*Contributed equally

Key words: breast cancer, METTL14, N6-methyladenosine, microRNA, RT-qPCR to be most enriched in cancer pathways, and DE miRNAs were enriched in cell adhesion terms. hsa-miR-146a-5p modulated by METTL14 promoted cell migration and invasion. METTL14 modulates m6A modification and hsa-miR-146a-5p expression, thereby affecting the migration and invasion of breast cancer cells.

\section{Introduction}

Breast cancer $(\mathrm{BC})$ is the most frequently diagnosed cancer and the leading cause of cancer-related mortality among women worldwide $(1,2)$. Despite the improved prognosis of individuals with $\mathrm{BC}$ and advances in radical surgery along with adjuvant therapy $(3,4), \mathrm{BC}$ is associated with common recurrence and persistently high mortality rates. BC frequently leads to multi-organ distant metastasis, such as in the lung, bone, liver, and brain (5). Studies are underway to identify therapeutic targets for BC, but more research in this area is needed.

Posttranscriptional modifications can influence the activity and stability of cellular RNAs, and the most abundant of these modifications is N6-methyladenosine (m6A) (6,7). In many species including higher eukaryotes, m6A modification is reported to be involved in mRNA splicing, translation, and stability; these activities also occur in rRNA and miRNA (8). m6A has been implicated in the development of numerous human diseases, including tumor formation (9). m6A methylation is controlled by the RNA methyltransferase complex (m6A 'writers'), RNA demethylases (m6A 'erasers'), and m6A readers. The m6A 'writer' is composed of Wilms tumor 1-associated protein (WTAP) (10), methyltransferase-like 3 and 14 (METTL3 and METTL14) (11), and protein virilizer homologue (KIAA1429), while heterogeneous nuclear ribonucleoprotein and YTH domain-containing RNA binding protein function as m6A 'readers' (12). AlkB homolog 5 (ALKBH5) and fat mass and obesity-associated protein (FTO) work as m6A 'erasers' to remove it from the target region $(13,14)$. Cross-talk and dynamic equilibrium among m6A writers, readers, and erasers play important roles in cancer development $(15,16)$.

Current efforts focusing on m6A and tumor development utilize advanced transcriptome-wide approaches for 
m6A sequencing. It has been shown that the METTL3 level is increased in lung carcinoma (LC) and modulates LC cell proliferation, survival, and invasion by regulating translation of target mRNA (17). Other research has revealed that FTO regulates the progression of cervical squamous cell carcinoma (CSCC) through mRNA demethylation (18). Additionally, ALKBH5 has been found to maintain the glioblastoma stem-like cell tumorigenicity through modulating FOXMI (19). Both METTL3 and METTL14 dramatically promote glioblastoma stem cell growth, self-renewal, and tumorigenesis (20). All of this evidence indicates a role for m6A and its enzyme system in tumor progression, but its downstream factors and signaling pathways are still unclear.

MicroRNAs (miRNAs) are a group of small non-coding RNAs, 21-24 nucleotides long, that are found in diverse organisms and regulate mRNA expression (21). Evidence indicates that m6A modification affects miRNA processing and expression: Altered METTL3/m6A may contribute to abnormal miRNA expression in many biological processes, especially in cancers (22). METTL3 is reported to be modulated by HBXIP by inhibiting miRNA let-7g in breast cancer (23). It has also been shown that METTL14 suppresses the metastasis of hepatocellular carcinoma by regulating m6A-dependent primary microRNA processing (8). In the present study, we aimed to identify the mechanism and function of METTL14/m6A in BC cells, as well as describe the potential effect of METTL14 on the miRNA expression profile and the effect of hsa-miR-146a-5p modulated by METTL14 on the function in BC cells.

\section{Materials and methods}

Study subjects and blood samples. We obtained preserved BC tissue samples and their adjacent normal breast tissues from Nanjing Hospital (Nanjing, Jiangsu, China); the BC patients underwent surgical treatment between January 2017 and January 2018. Demographic and clinicopathological information for these patients is documented in Table I. This study was approved by the Human Research Ethics Committee of Nanjing Medical University (Nanjing, Jiangsu, China). All patients gave their written informed consent according to the Declaration of Helsinki, and all experimental procedures were conducted following its guidelines.

RNA m6A quantification. Total RNA was extracted from tissues using TRIzol (Invitrogen; Thermo Fisher Scientific, Inc.) following the manufacturer's manual. RNA quality was analyzed by NanoDrop (Thermo Fisher Scientific, Inc.) and $1 \%$ agarose gel electrophoresis. The EpiQuik ${ }^{\mathrm{TM}}$ m6A RNA Methylation Quantification Kit (P-9005-48, Colorimetric, Epigentek, USA) was applied to analyze the m6A content in high-quality RNAs. Briefly, 100-300 ng RNA was loaded into test wells. Capture and detection antibody solutions were then added to each test well separately in an appropriately diluted concentration following the manufacturer's protocol. m6A levels were determined according to the absorbance of each sample at $450 \mathrm{~nm}$, and a standard curve was then used to calculate relative m6A content.

Western blot analysis. Protein samples were taken from the cells lysed using SDS (Beyotime). Total protein was determined using the Pierce BCA assay kit (Thermo Fisher Scientific, Inc.). SDS-PAGE gels (10\%) were used to separate the different proteins, and $15 \mu \mathrm{g}$ total protein was loaded per lane. Then, all protein bands were visible on polyvinylidene fluoride (PVDF) membranes (Millipore). The membranes were immersed in primary antibodies, anti-METTL14 (dilution 1:1,000; cat. no. Ab220030; Abcam) and anti-GAPDH (dilution 1:1,000; cat. no. AF0006; Beyotime Institute of Biotechnology) for $1 \mathrm{~h}$ at room temperature. Next, the membranes were washed and incubated with HRP-conjugated goat anti-rabbit IgG (dilution 1:1,000; cat. no. A0216; Beyotime Institute of Biotechnology) for $2 \mathrm{~h}$. Protein bands were visualized using ECL chemiluminescence reagent (Thermo Fisher Scientific, Inc.) and images were captured using the ChemiDoc MP system (Bio-Rad Laboratories, Inc.). Immunoblot data were normalized to GAPDH levels using ImageJ software (version 1.8.0; National Institutes of Health, Bethesda, MD, USA).

Cell culture and transfection. BC cell lines including MCF-7 and MDA-MB-231 were obtained from Zhongqiao Xinzhou Biotechnology Co. Ltd. Cells were sub-cultured in Dulbecco's modified Eagle's medium (DMEM) (Zhongqiao Xinzhou Biotechnology Co. Ltd.) mixed with $10 \%$ Gibco fetal bovine serum (FBS) (Thermo Fisher Scientific, Inc.), $100 \mathrm{mg} / \mathrm{ml}$ streptomycin (Procell), and $100 \mathrm{U} / \mathrm{ml}$ penicillin at $37^{\circ} \mathrm{C}$ in a humidified atmosphere with $5 \% \mathrm{CO}_{2}$. When the cell density reached $80-90 \%$, siRNAs (listed in Table II), hsa-miR-146a-5p inhibitor and plasmids, purchased from GenePharma, were transfected using Lipofectamine 3000 reagent (Thermo Fisher Scientific, Inc.) into MCF-7 and MDA-MB-231 cells, respectively. In addition, 3-deazaadenosine (m6A inhibitor) was purchased from Sigma-Aldrich (Merck KGaA). All procedures followed the manufacturer's instructions.

Cell function as assessed by Transwell assay. For the cell migration assay, $1 \times 10^{5} \mathrm{MCF}-7$ or MDA-MB-231 cells were loaded into the upper chamber inserts (Millipore) with $500 \mu \mathrm{l}$ of serum-free DMEM. The lower chambers contained $700 \mu \mathrm{l}$ DMEM with $10 \%$ FBS. After 24-48 h, the cells underneath the membrane were stained with $800 \mu \mathrm{l}$ crystal violet (cat. no. C0121; Beyotime Institute of Biotechnology) at room temperature for $30 \mathrm{~min}$ and counted using a light microscope in 3 random high-power fields. For the invasion experiment, chambers precoated with matrix adhesive (Corning, USA) were applied. All experiments were repeated three times.

CCK-8 assay. Cell proliferation abilities of the MCF-7 or MDA-MB-231 cells were evaluated by Cell Counting Kit- 8 assays. Cells were transfected as mentioned above. Post-transfection at $48 \mathrm{~h}$, the cells were digested, resuspended into single-cell suspension, seeded into 96 -well plates with $100 \mu \mathrm{l}$ of cells per well $\left(2 \times 10^{4} \mathrm{cell} / \mathrm{ml}\right)$, and incubated at $37^{\circ} \mathrm{C}$ with $5 \% \mathrm{CO}_{2}$. CCK-8 reagent $(10 \mu \mathrm{l})$ was added to per well after incubation at $0,24,48,72$ and $96 \mathrm{~h}$, and incubated for $1 \mathrm{~h}$ for each time point. Absorbance was measured via a microplate reader (Bio-Rad Laboratories) at $450 \mathrm{~nm}$.

Small RNA library construction and sequencing. Total RNA was extracted from the MCF-7 and MDA-MB-231 cells, and then quantitated and purified. RNAs of high quality were used 
Table I. Demographic and clinicopathological information of the 58 BC patients.

\begin{tabular}{|c|c|c|c|c|}
\hline \multirow[b]{2}{*}{ Characteristics } & \multirow[b]{2}{*}{ No. of patients } & \multicolumn{2}{|c|}{ METTL14 expression } & \multirow[b]{2}{*}{ P-value } \\
\hline & & Low n (\%) & High n (\%) & \\
\hline Age (years) & & & & 0.5992 \\
\hline$<60$ & 28 & $15(53.5)$ & $13(46.5)$ & \\
\hline$\geq 60$ & 30 & $14(46.7)$ & $16(53.3)$ & \\
\hline Typing & & & & 0.9389 \\
\hline Luminal A & 4 & $2(50)$ & $2(50)$ & \\
\hline Luminal B & 33 & $16(39.4)$ & $17(60.6)$ & \\
\hline HER-2 ${ }^{+}$ & 9 & $5(55.6)$ & $4(44.4)$ & \\
\hline Basal-like & 12 & $5(41.7)$ & $7(58.3)$ & \\
\hline Microvascular invasion & & & & 0.4297 \\
\hline Yes & 27 & $12(44.4)$ & $15(55.6)$ & \\
\hline No & 31 & $17(54.8)$ & $14(45.2)$ & \\
\hline Nerve invasion & & & & 0.6489 \\
\hline Yes & 15 & $7(46.7)$ & $8(53.3)$ & \\
\hline No & 43 & $23(53.5)$ & $20(46.5)$ & \\
\hline TNM & & & & $0.0483^{\mathrm{a}}$ \\
\hline $\mathrm{T} 1+\mathrm{T} 2$ & 54 & $30(55.6)$ & $24(44.4)$ & \\
\hline $\mathrm{T} 3$ & 4 & 0 & $4(100)$ & \\
\hline Pathological stage & & & & 0.0827 \\
\hline $\mathrm{I}+\mathrm{II}$ & 47 & $22(46.8)$ & $25(53.2)$ & \\
\hline III & 11 & $2(18.2)$ & $9(81.8)$ & \\
\hline WHO & & & & 0.0768 \\
\hline $\mathrm{I}+\mathrm{II}$ & 27 & $12(44.4)$ & $15(55.6)$ & \\
\hline III & 31 & $7(22.6)$ & $24(77.4)$ & \\
\hline Metastasis & & & & 0.2389 \\
\hline $\mathrm{pN} 0+\mathrm{pN} 1$ & 47 & $22(46.8)$ & $25(53.2)$ & \\
\hline $\mathrm{pN} 2+\mathrm{pN} 3$ & 11 & $3(27.3)$ & $8(72.7)$ & \\
\hline
\end{tabular}

${ }^{\mathrm{a}} \mathrm{P}<0.05$, represents a significant difference. BC, breast cancer; METTL14, methyltransferase like 14 .

to establish small RNA libraries with the NEBNext ${ }^{\circledR}$ Multiplex Small RNA Library Prep Kit following the manufacturer's instructions (New England Biolabs). A Hiseq2500 (Illumina, USA) was then used for small RNA sequencing.

Data filtering and mapping. The raw data were filtered and optimized with FastQC (http://www.bioinformatics. babraham.ac.uk/projects/fastqc/) (24) to discard short ( $<15 \mathrm{nt})$ and low-quality reads. The small RNA clean reads were then matched to the miRBase21.0 (http://www.mirbase.org/) (25) to screen out known miRNAs (20-25 bp), and unmapped reads were further scheduled to piRNAcluster (http://www. smallrnagroup.uni-mainz.de/piRNAclusterDB.html). The matched reads were then compared with the National Center for Biotechnology Information database (https://www.ncbi. nlm.nih.gov/) to find known piRNAs. Unmapped reads were then assigned to Genomic tRNA Database (GtRNAdb, http://gtrnadb.ucsc.edu/) and tRFdb (http:/genome.bioch. virginia.edu/trfdb/) to define tRNA derived fragments (tRFs). Finally, the remaining unmatched reads were allocated to
Rfam (http://rfam.xfam.org/) to identify small nucleolar RNA (snoRNA) as well as small nuclear RNA (snRNA) sequences. All identifiable small RNAs were separated and defined.

Differentially expressed miRNA identification and target prediction. Differentially expressed (DE) miRNAs were filtered with Ebseq2.0 packages (26). $\mid \log _{2}$ (fold change) I $>1$ and false discovery rate (FDR) $<0.05$ were considered to be significantly different. Miranda and RNAhybrid were applied to determine DE miRNAs' target mRNAs (27). mRNAs that met both Miranda (score $\geq 150$ and energy <-20) and RNAhybrid (energy <-25) cutoffs were considered to be the assumed DE mRNAs. Finally, the miRNA/mRNA interaction network was diagrammed using Cytoscape software (28) (version 3.5.1).

Functional enrichment analysis of target genes. Target genes screened using the database for Annotation, Visualization, and Integrated Discovery 6.8 Bioinformatics Tool (DAVID 6.8) (29) were then submitted for Gene Ontology (GO, http://www.geneontology.org/) (30) analysis and Kyoto 
Table II. Primers and sequences.

\begin{tabular}{ll}
\hline Primers & \multicolumn{1}{c}{ Sequences (5'-3') } \\
\hline METTL14-F & AAATGCTGGACTTGGGATGATA \\
METTL14-R & CCCATTTTCGTAAACACACTCTT \\
METTL3-F & GAACACTGCTTGGTTGGTGTCA \\
METTL3-R & TGGAACGAACCTCAGCTACGAT \\
WTAP-F & TTCCCAAGAAGGTTCGATTGAG \\
WTAP-R & AGACTCCTGCTGTTGTTGCTTTAG \\
KIAA1429-F & CGATAACTTGATGACCCCAGAA \\
KIAA1429-R & ATAACGGCAAGATTCCATTTC \\
hsa-miR-146a-5p-F & GGCGGTGAGAACTGAATTCC \\
hsa-miR-29b-3p-F & CGCAGTAGCACCATTTGAAAT \\
hsa-miR-3691-5p-F & CGCAGAGTGGATGATGGAGAC \\
hsa-miR-221-3p-F & GGCGGAGCTACATTGTCTGC \\
hsa-miR-4326-F & GGGCGGTGTTCCTCTGTCT \\
all-miR-R & AGTGCGTGTCGTGGAGTCG \\
B2M-F & CTCTTTCTGGCCTGGAGGCTAT \\
B2M-R & AGTCAACTTCAATGTCGGATGGAT \\
U6-F & CGATACAGAGAAGATTAGCATGGC \\
U6-R & AACGCTTCACGAATTTGCGT \\
Si-METTL14 & UCUUAUCCAACCUUUCUUCCG \\
\hline
\end{tabular}

METTL14, methyltransferase-like 14; WTAP, Wilms tumor 1-associated protein; KIAA1429, protein virilizer homologue; F, forward; R, reverse.

Encyclopedia of Genes and Genomes (KEGG, http://www. kegg.jp/) pathway enrichment analysis (31). GO analyses were divided into cellular component (CC), biological process (BP), and molecular function (MF) analysis. KEGG pathway enrichment analysis was conducted to explore the biological pathways in which the DE miRNA target mRNAs were potentially involved. The cutoff threshold was set to P-value $<0.05$.

METTL14 and miRNA expression as verified by real-time quantitative PCR (RT-qPCR). Total RNA was reverse-transcribed into complementary DNA (cDNA) following the manufacturer's instructions (ThermoScientific ${ }^{\mathrm{TM}}$ RevertAid $^{\mathrm{AM}}$ First Strand cDNA Synthesis kit, cat. \#K1622; Thermo Fisher Scientific, Inc.). qPCR was conducted using the ABI Q6 detection system (Applied Biosystems Inc.; Thermo Fisher Scientific, Inc.). The delta-delta $\mathrm{Cq}$ method (2- $\left.{ }^{-\Delta \Delta \mathrm{Cq}}\right)$ (32) was used to measure the relative content of the miRNAs. Primers were ordered from Sangon Biotech, and primer sequences are displayed in Table II. B2M and U6 were used as the internal control for Mettll4 and miRNA, respectively.

Statistical analysis. SPSS 19.0 (IBM Corp.) was used to perform statistical analysis. The P-values of the clinical indexes were calculated using Chi-square test. The paired Student's t- test was used for METTL14 level in 20 paired BC and control tissues. The unpaired Student's t-test was applied for two-group comparison of the other assays, and one-way analysis of variance (ANOVA) followed by Tukey's multiple comparison test was used when more than two groups were evaluated. P-value $<0.05$ was considered as indicative of a statistically significant difference.

\section{Results}

m6A methyltransferase METTL14 (also known as methyltransferase like 14 and N6-adenosine-methyltransferase non-catalytic subunit) is aberrantly expressed in BC tissues. Evidence indicates that there is an aberrant N6-methyladenosine level in BC (23). We assumed that abnormal m6A modification in $\mathrm{BC}$ was due to abnormal expression of m6A methyltransferases. To test this hypothesis, the mRNA levels of METTL3, METTL14, WTAP, and KIAA1429 in 20 paired BC and paracancerous tissue samples were determined by qPCR. Results revealed that METTL14 expression was significantly increased in $\mathrm{BC}$, while no significant difference was observed in the expression of METTL3, WTAP and KIAA1429 (Fig. 1A). Moreover, western blot analysis showed that the METTL14 protein level was significantly increased in BC tissues compared to that in the normal tissue (Fig. 1B). After we analyzed the demographic and clinicopathological information of the BC patients in Table I, we found that there was no difference between the METTL14 low and high expression samples with regard to age, typing, microvascular invasion, nerve invasion status, pathological stage, WHO stage or metastasis status, while METTL14 expression was related to TNM grade.

METTL14 modulates the m6A modification in BC cells. METTL14 has been reported to regulate m6A formation; therefore, we inferred that aberrant METTL14 expression may play an important role in m6A modifications in BC cells. We transfected METTL14 siRNA into MCF-7 and MDA-MB-231 cells to create METTL14 knockdown cell lines (MCF-7 si-METTL14 and MDA-MB-231 si-METTL14; Fig. 2A), and we cloned METTL14 into the pcNDA3.1 vector to achieve overexpressing cell lines (MCF-7 pc-METTL14 and MDA-MB-231 pc-METTL14; Fig. 2B). As expected, METTL14 overexpression increased m6A levels, and knockdown resulted in decreased m6A levels in the MDA-MB-231 (Fig. 2C and D). Consistently, METTL14 overexpression increased m6A levels, and knockdown resulted in decreased m6A levels in the MCF7 cells (Fig. 2E and F). After METTL14 overexpression and knockdown, the obtained results revealed that METTL14 upregulated the m6A levels in MDA-MB-231 and MCF7 cells. Therefore, METTL14 modulates the m6A modification in BC.

Through regulation of $m 6 A$ modification METTL14 enhances migration and invasion capacity of $B C$ cells. We next assessed the effect of the aberrant expression of METTL14 on BC cell migration and invasion. The BC cells were subsequently transfected with METTL14 overexpression or control vector for functional assays. METTL14 overexpression promoted the migration and invasion of the MDA-MB-231 and MCF-7 cells (Fig. 3). Moreover, it was confirmed that the m6A inhibitor significantly inhibited the m6A level in the MDA-MB-231 cells (Fig. 4A). With the m6A inhibitor treatment, the enhanced effect of METTL14 on cell migration and invasion was suppressed (Fig. 4B and C), indicating that METTL14 may act as a positive regulator of BC function. We also tested the effect of METTL14 on cell proliferation using the CCK-8 assay. Results revealed that 
A
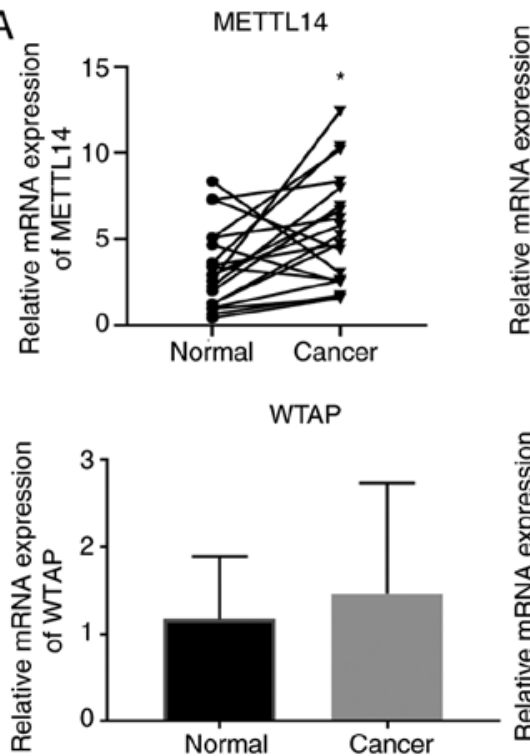

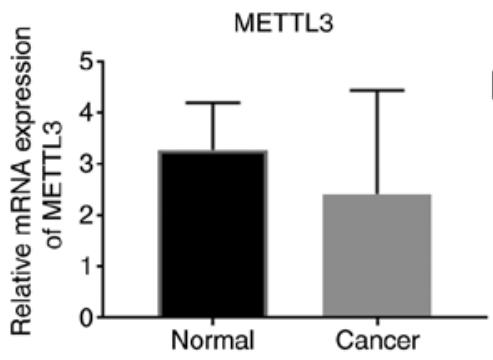

KIAA1429

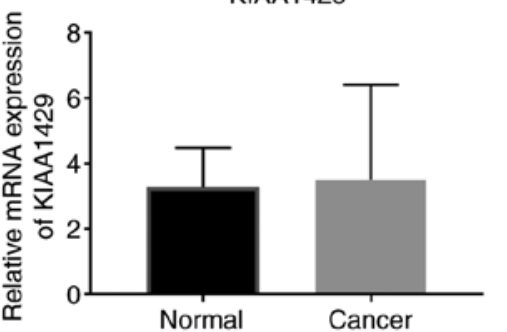

B

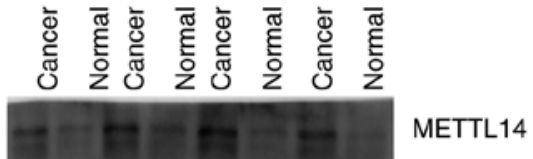

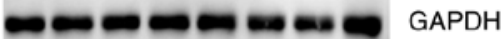

Figure 1. Aberrant expression of METTL14 in BC tissues. (A) The mRNA level of METTL3, METTL14, WTAP and KIAA1429 in 20 paired BC and control tissues was determined by qPCR. (B) The protein level of METTL14 in 7 pairs of BC/adjacent tissues was determined by western blot analysis. The results are presented as the means \pm SD. ${ }^{*} \mathrm{P}<0.05$. BC, breast cancer; METTL3 and METTL14, methyltransferase-like 3 and 14; WTAP, Wilms tumor 1-associated protein.

A
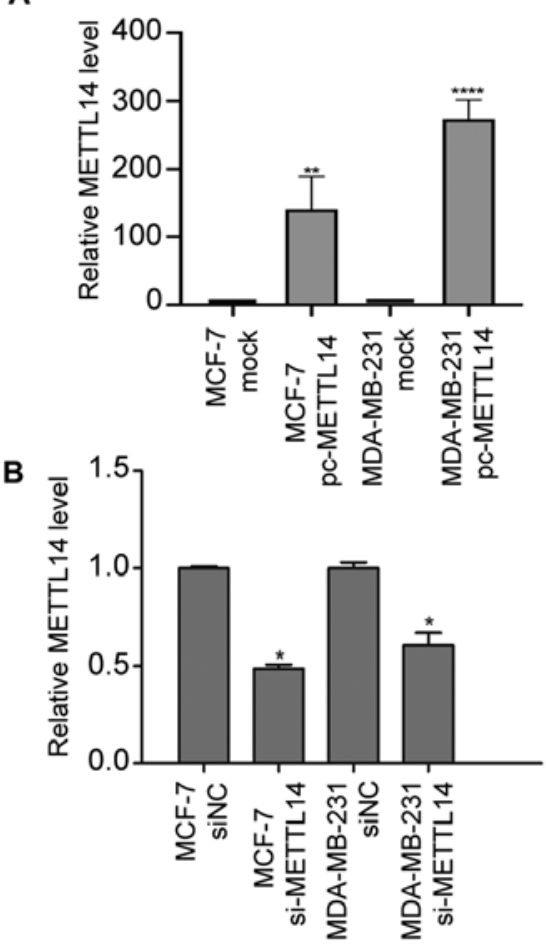

C
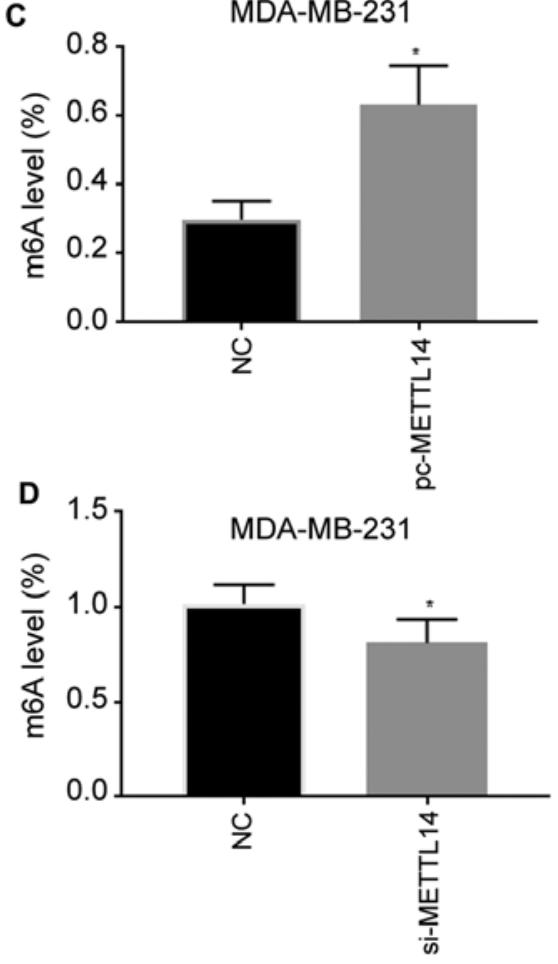

E
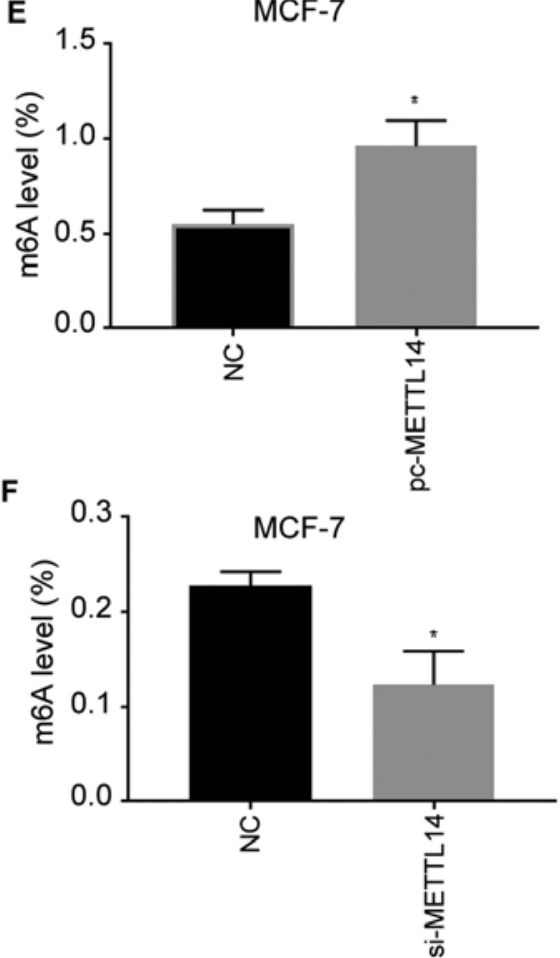

Figure 2. METTL14 interference and overexpression affect m6A levels in BC cell lines. (A and B) Overexpression and interference efficiency of METTL14 were determined in MDA-MB-231 and MCF-7 cells by RT-qPCR. The effect of METTL14 overexpression (C and E) and knockdown (D and F) on the m6A level was measured using the m6A RNA Methylation Quantification Kit in MDA-MB-231 and MCF-7 cells. The results are presented as the mean \pm standard error of the mean (n=3). ${ }^{*} \mathrm{P}<0.05,{ }^{* * *} \mathrm{P}<0.01$ and ${ }^{* * * * *} \mathrm{P}<0.0001$. METTL14, methyltransferase-like 14; BC, breast cancer; m6A, N6-methyladenosine. MCF-7 pc-METTL14 and MDA-MB-231 pc-METTL14, METTL14-overexpressing cell lines; MCF-7 si-METTL14 and MDA-MB-231 si-METTL14, METTL14-knockdown cell lines; RT-qPCR, reverse transcription-quantitative real-time PCR.

METTL14 gain-of-expression had little effect on cell proliferation in the MCF-7 and MDA-MB-231 cells (Fig. S1).

METTL14 overexpression reshapes the miRNA profiling of BC cells. To explore the effect of abnormal expression of METTL14 on miRNA processing and expression, we conducted miRNA sequencing on METTL14 overexpression and mock groups of MCF-7 and MDA-MB-231 cells. In cells overexpressing METTL14, the number of upregulated miRNAs was higher than that of the downregulated miRNAs in both cell lines 
A
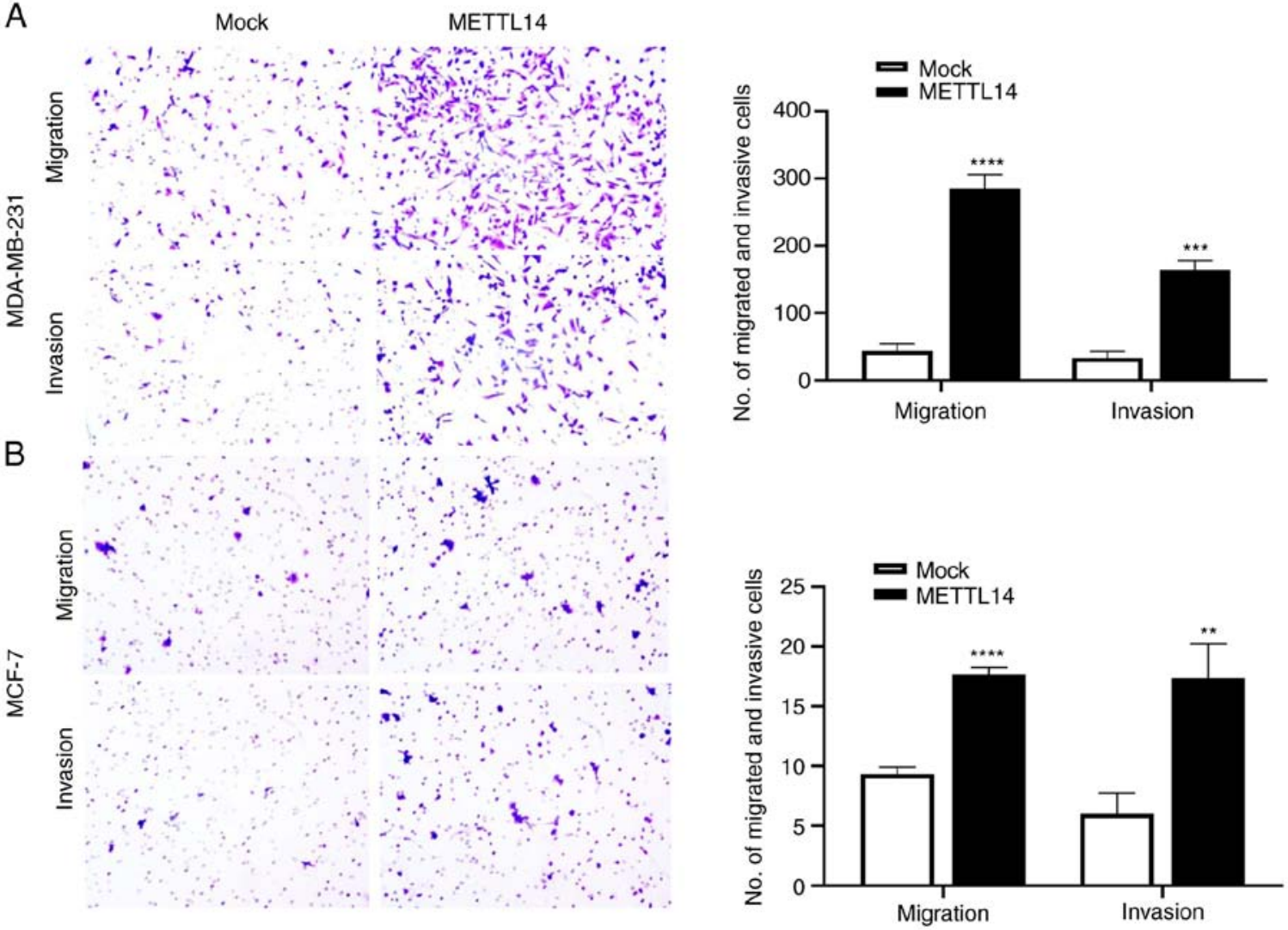

Figure 3. METTL14 overexpression promotes BC cell migration and invasion. (A) METTL14 overexpression promotes migration and invasion in MDA-MB-231 cells. (B) METTL14 overexpression promotes migration and invasion in MCF-7 cells. The images (magnification, x20) were captured using a light microscope. Values are indicated as mean \pm SD from three independent experiments. ${ }^{* *} \mathrm{P}<0.01,{ }^{* * * *} \mathrm{P}<0.001$ and ${ }^{* * * *} \mathrm{P}<0.0001$. METTL14, methyltransferase-like 14 ; $\mathrm{BC}$, breast cancer.

A
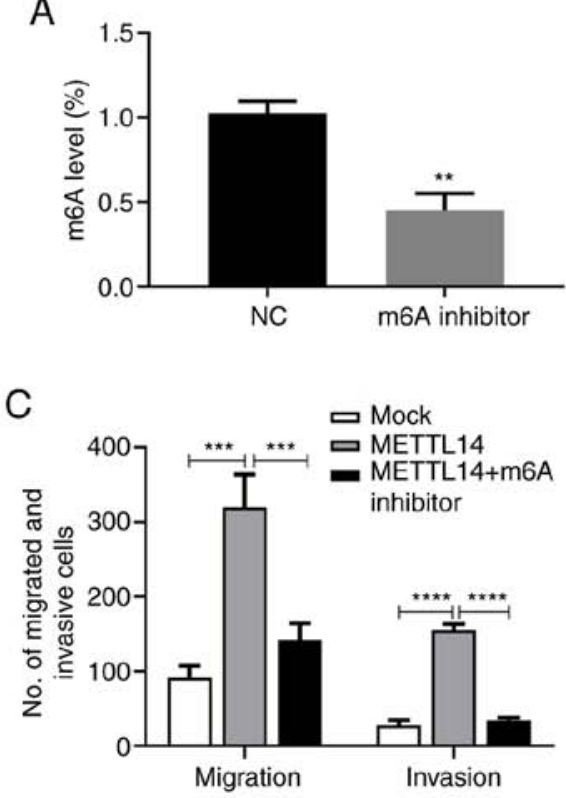

B

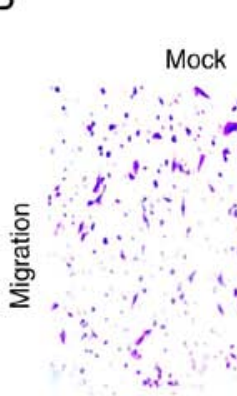

MDA-MB-231

METTL14

METTL14+ m6A inhibitor

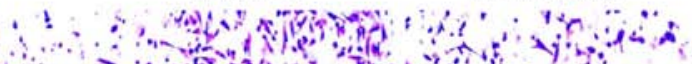
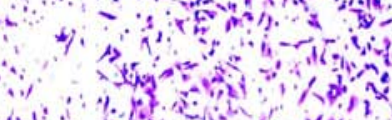

(iv) $v+8$

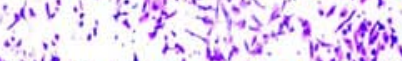

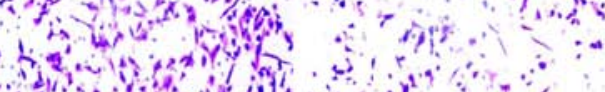
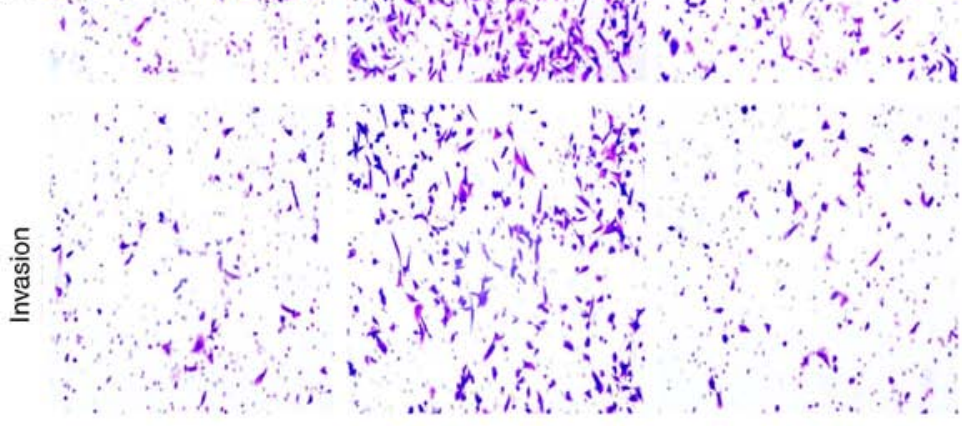

Figure 4. METTL14 promotes migration and invasion of BC cells by regulating the m6A level. (A) Effect of m6A inhibitor on the m6A level was measured using the m6A RNA Methylation Quantification Kit in MDA-MB-231 cells. (B) METTL14 overexpression promoted migration and invasion in MDA-MB-231 cells, while treatment with an m6A inhibitor suppressed the enhanced cell migration and invasion. (C) Migration and invasive abilities of the BC cells were analyzed. Data are the means \pm SDs $(n=3) .{ }^{* *} \mathrm{P}<0.01,{ }^{* * *} \mathrm{P}<0.001$ and ${ }^{* * * *} \mathrm{P}<0.0001$. METTL14, methyltransferase-like 14; BC, breast cancer; m6A, N6-methyladenosine.

(Fig. 5A and B). Specifically, 685 miRNAs were upregulated and only 65 were downregulated in the MDA-MB-231 cells, and
52 miRNAs were upregulated and 14 were downregulated in the MCF-7 cells. Moreover, 34 DE miRNAs showed the same 

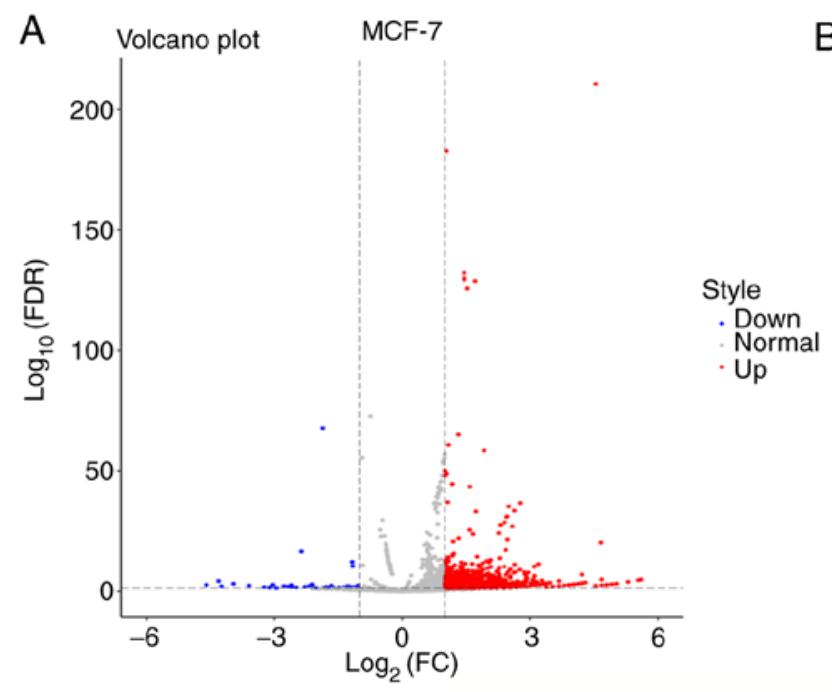

B
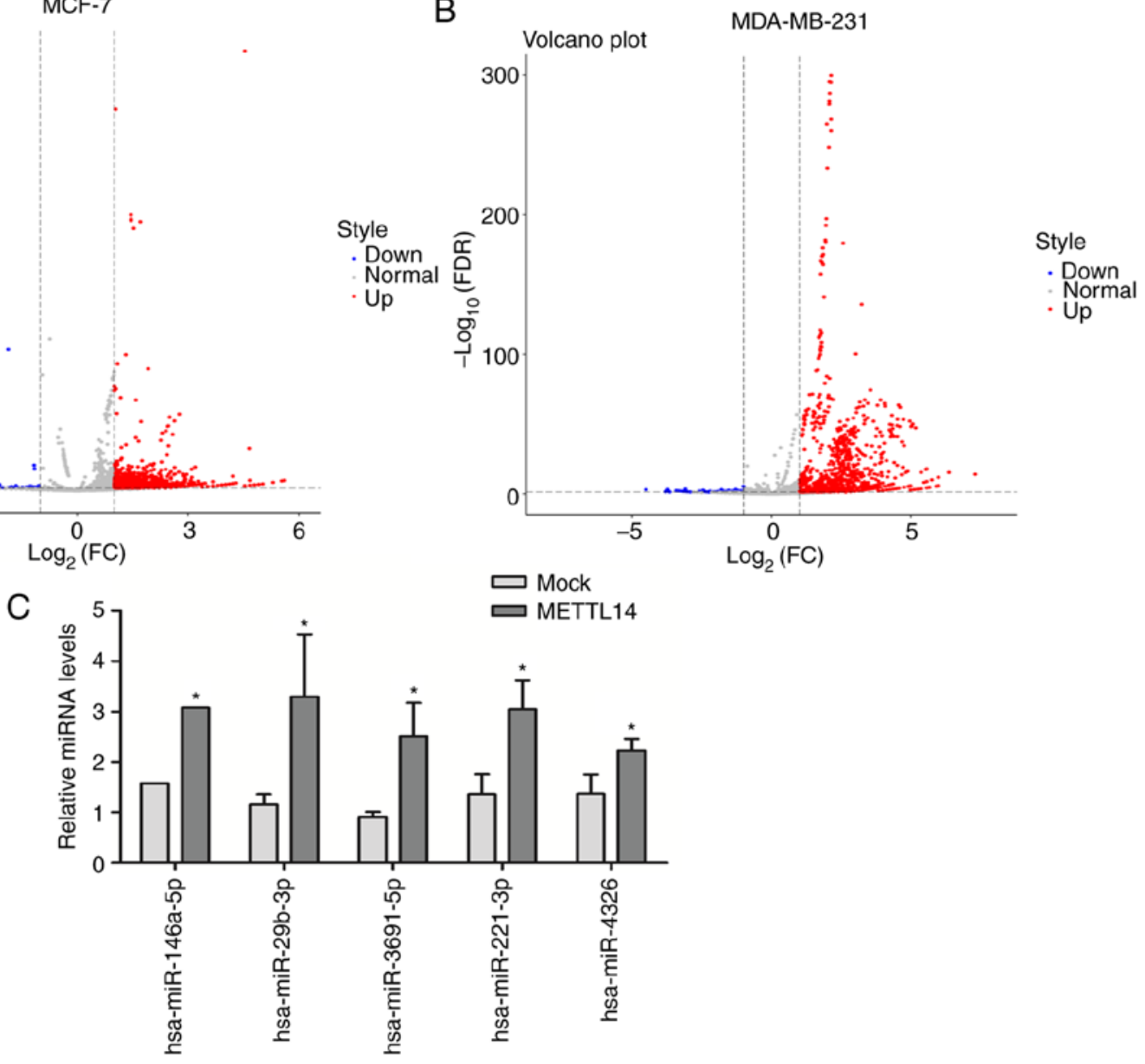

Figure 5. METTL14 overexpression affects the miRNA profile. (A and B) Volcano plots show the significantly different miRNAs induced by METTL14 overexpression in MCF-7 and MDA-MB-231 cells. Red dots and blue dots represent the upregulated and downregulated miRNAs, respectively. The log2 (fold change) was plotted against the $-\log 10$ (P-value). (C) Expression levels of 5 miRNAs were verified by RT-qPCR. Values are indicated as mean \pm SD from three independent experiments. ${ }^{*} \mathrm{P}<0.05$. METTL14, methyltransferase-like 14.

upregulated/downregulated tendency between the MCF-7 and MDA-MB-231 cell lines with METTL14 overexpression; of these, 33 miRNAs were upregulated and 1 miRNA was downregulated. Expression of 5 miRNAs including hsa-miR-146a-5p, hsa-miR-29b-3p, hsa-miR-3691-5p, hsa-miR-221-3p, and hsa-miR-4326 was verified by RT-qPCR (Fig. 5C), and the results of these expression levels of miRNAs were in accordance with miRNA sequencing data.

Functional enrichment and miRNA-mRNA interaction network analysis reveal the DE miRNAs targeting BC development. To evaluate the DE miRNA targeting mRNAs in the MCF-7 and MDA-MB-231 cells during METTL14 overexpression, GO and KEGG pathway enrichment analyses were performed. The target genes of DE miRNAs were mainly enriched in 'regulation of transcription', 'cell adhesion', 'protein phosphorylation', 'phosphorylation', 'axon guidance', 'nervous system development', and 'neurotrophin TRK receptor signaling pathway' in both the MCF-7 and MDA-231 cell lines. In addition, 'angiogenesis', 'cell differentiation' and 'negative regulation of cell proliferation', which are related to $\mathrm{BC}$ development, were also enriched in the TOP20 GO terms (Fig. 6A and B). Moreover, KEGG pathway enrichment analysis revealed that the DE miRNAs target mRNAs enriched in KEGG terms such as 'MAPK signaling pathway', 'Pathways in cancer', 'Ras signaling pathway', 'PI3K-Akt signaling pathway', 'Focal adhesion', 'Wnt signaling pathway', and 'Rap1 signaling pathway' were also closely related to the occurrence and development of cancer (Fig. 6C and D). All GO and KEGG analyses indicated that aberrant METTL14 expression altered the miRNA expression profile, adjusting and controlling BC cell activities. We utilized Cytoscape software to depict an integrated mRNA/miRNA interaction network that included 30 upregulated miRNAs (Fig. 7A) and 2 downregulated (Fig. 7B) miRNAs and their targets involved in 'Pathways in cancer'. In this complex network, upregulated hsa-miR-149-3p and downregulated hsa-554b-3p were shown to have more targets that related to the cancer pathway. In addition, hsa-miR-146a-5p, hsa-miR-29b-3p and hsa-miR-3691-5p appeared in the network, and their expression was confirmed by RT-qPCR.

hsa-miR-146a-5p modulated by METTL14 promotes migration and invasion capacity of $B C$ cells. We next evaluated the effect of hsa-miR-146a-5p modulated by METTL14 on migration and invasion of BC cells. First, we confirmed that the hsa-miR-146a-5p inhibitor significantly inhibited hsa-miR-146a-5p expression in the MDA-MB-231 cells (Fig. 8A). The METTL14 overexpression vector and 
A

(MCF-7)

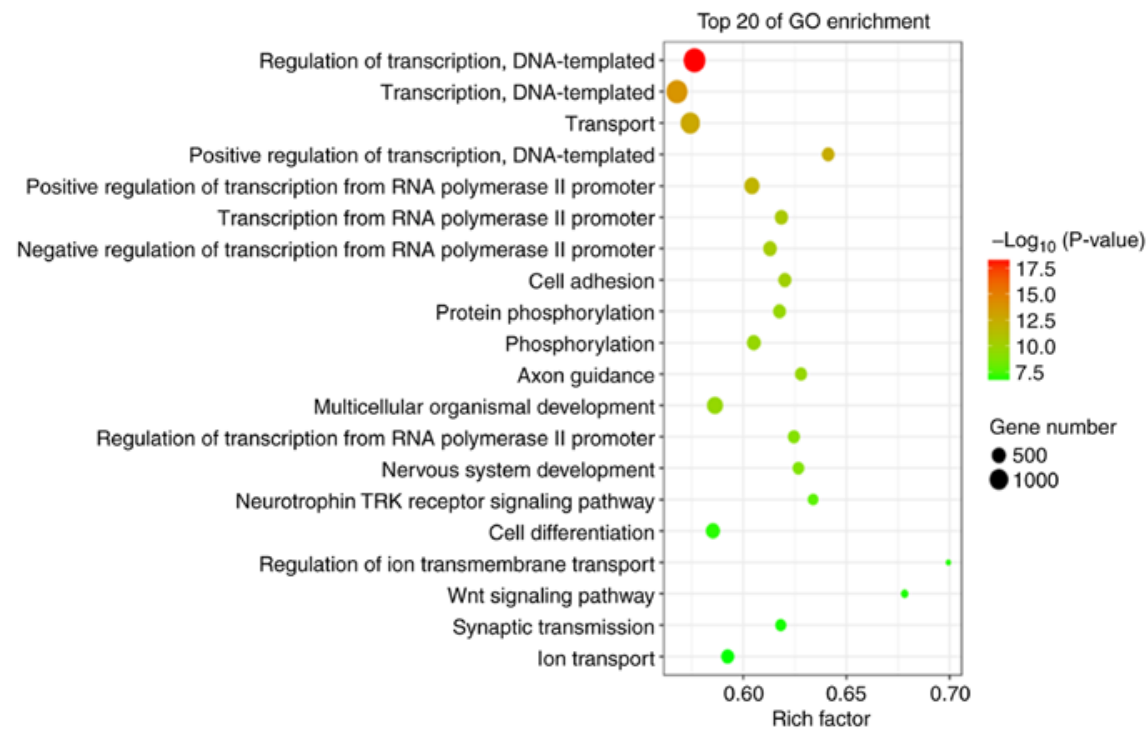

B

(MDA-MB-231)

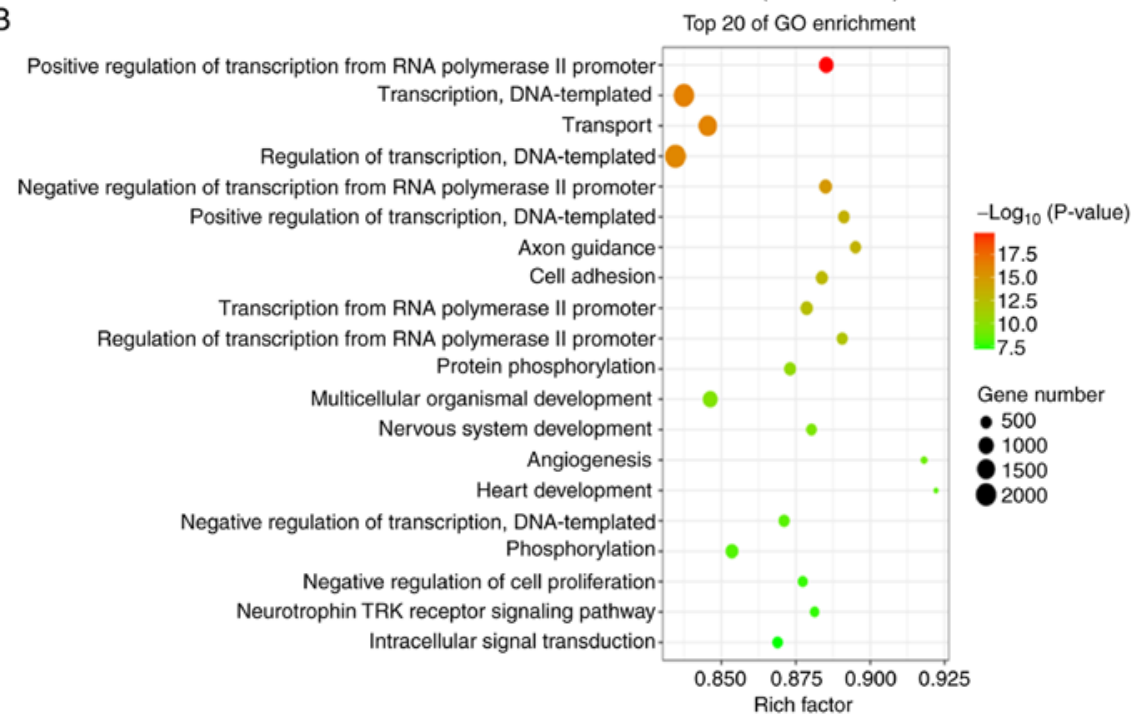

C

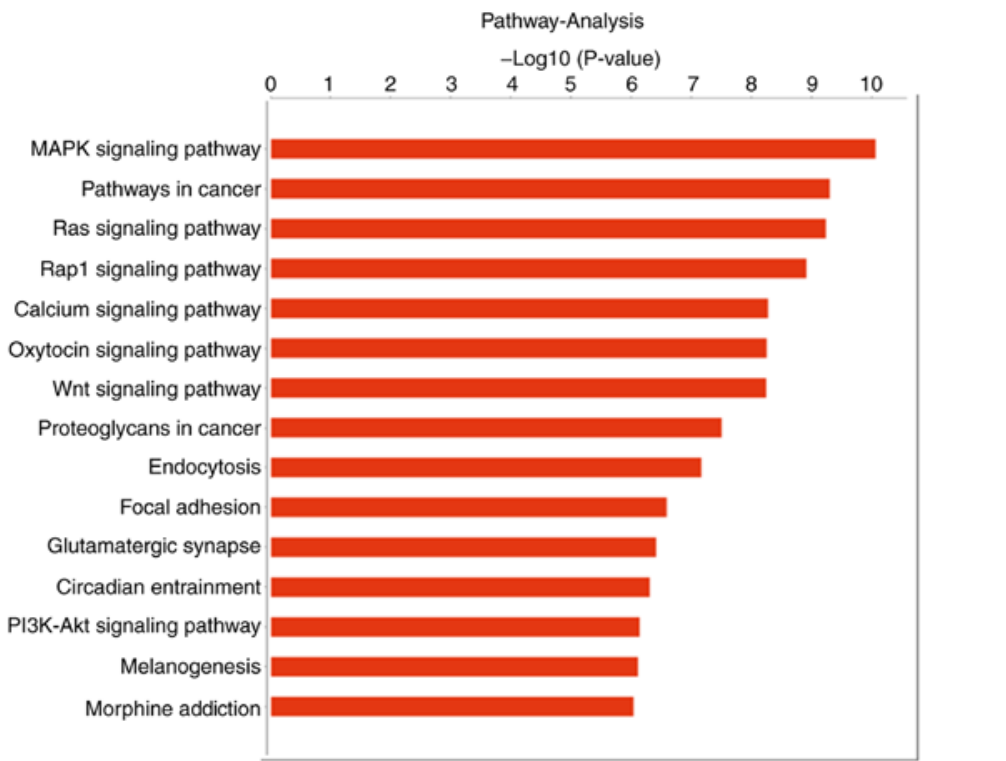

Figure 6. Functional enrichment. Gene Ontology (GO) functional enrichment analysis for predicted target genes of DE miRNAs in (A) MCF-7 and (B) MDA-MB-231 cells. The x-axis refers to the enrichment factor including gene numbers and -log10 (P-value), and the y-axis refers to the GO terms. Kyoto Encyclopedia of Genes and Genomes analyses (KEGG) functional enrichment analysis for predicted target genes of DE miRNAs in (C) MCF-7. 


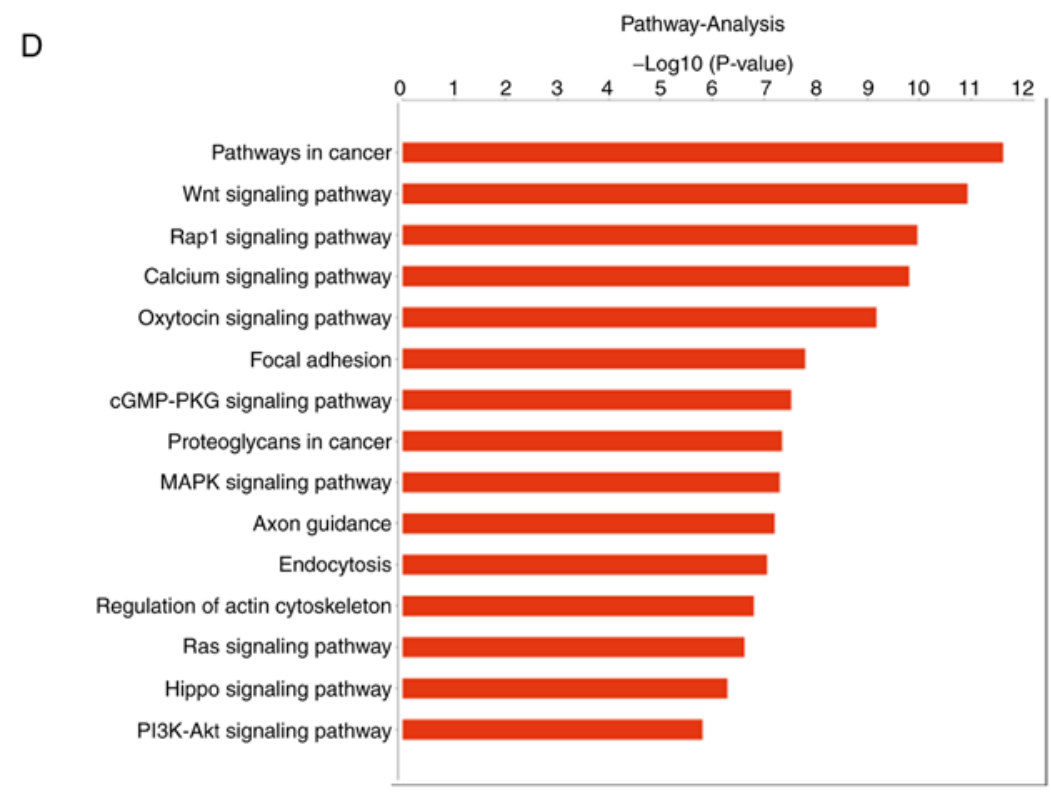

Figure 6. Continued. Kyoto Encyclopedia of Genes and Genomes analyses (KEGG) functional enrichment analysis for predicted target genes of DE miRNAs in (D) MDA-MB-231 cells. The x-axis refers to the number of genes, and the y-axis refers to KEGG pathway terms. The color represents the P-value. DE, differentially expressed.

hsa-miR-146a-5p inhibitor as well as the negative control were subsequently transfected into MDA-MB-231 cells. Overexpression of METTL14 promoted the migration and invasion capacity of MDA-MB-231 cells, while treatment with the hsa-miR-146a-5p inhibitor suppressed this enhanced cell migration and invasion ability (Fig. $8 \mathrm{~B}$ and C).

\section{Discussion}

Early studies have shown that the epigenetic regulation of N6-methyladenosine (m6A) is involved in many post-transcriptional regulatory processes, similarly to DNA histone modifications. Aberrant m6A is usually associated with the progression of disease and cancer. The present study is the first to show that aberrant expression of RNA methyltransferase METTL14 (methyltransferase-like 14) modulates the development and the level of m6A in breast cancer (BC), and to reveal that microRNA processing and expression is determined in a N6-methyladenosine-dependent manner. This introduces the idea that the relationship between $\mathrm{m} 6 \mathrm{~A}$ modification and miRNA processing is important in BC development.

With the development of whole-transcriptome m6A sequencing approaches, the functions of m6A RNA modification in cancer biology have been rapidly elucidated. RNA methyltransferase METTL3 or METTL14 and RNA demethylase fat mass and obesity-associated protein (FTO) are critical for glioblastoma stem cell (GSC) self-renewal and tumorigenesis (20). METTL3 promotes bladder cancer proliferation and metastasis via $\mathrm{AFF} 4 / \mathrm{NF}-\kappa \mathrm{B} / \mathrm{MYC}$ signaling (33). Additionally, $70 \%$ of endometrial tumors exhibit downregulated m6A methylation that may be due to METTL14 mutation or reduced METTL3 expression; these abnormalities in RNA methyltransferases promote proliferation and tumorigenicity of endometrial cancer via $A K T$ pathway activation (16). Reductions in m6A methylation lead to decreased expression of the negative $A K T$ regulator $P H L P P 2$ and increased expression of the positive $A K T$ regulator
mTORC2. However, the expression of RNA methyltransferase in BC, as well as its role and mechanism in cancer development, remain unclear. Our study revealed for the first that aberrant METTL14 expression is related to BC cell migration and invasion. We also tested the overexpression of METTL14 on BC cell growth using the CCK-8 assay, but METTL14 gain-of-expression had little effect on cell proliferation in MCF-7 cells. As shown in the Gene Ontology (GO) functional analysis, differentially expressed (DE) miRNAs were enriched in cell adhesion terms in both MCF-7 and MDA-MB-231 cells. Metastasis formation is a complex process, and requires the epithelial-mesenchymal transition (EMT) (34). During EMT, cell-to-cell and cell-to-matrix adhesion molecules are crucial for cells to break free from the primary tumor mass and enter the bloodstream to cause metastasis formation (35). Here, this evidence indicates that METTL14 may control BC cell metastasis by regulating miRNA levels that affect these adhesion molecules, and should be further verified in vitro and in vivo. This will be our future research direction.

Studies have shown that m6A modification is involved in transcriptome regulation in disease and cancer states. m6A modification has potential implications for miRNA biogenesis, as well as for mRNA-miRNA interactions (36). For instance, METTL14 is associated with hepatocellular carcinoma metastasis, and its depletion was found to inhibit miR-126 expression by modulating DGCR8 binding in an m6A-dependent manner (37). In our results, we were surprised to find that METTL14 overexpression led to increased expression of most miRNAs, especially in the cancer-related pathway-miRNA-mRNAs interaction network: 30 miRNAs were upregulated, and 2 miRNAs were downregulated. We hypothesize that increased $\mathrm{m} 6 \mathrm{~A}$ expression may promote pri-miRNA processing, thus leading to increased miRNA expression; this lays the groundwork for future studies.

The miRNA-mRNA interaction network that we investigated is related to the cancer pathway. hsa-miR-146a-5p, 


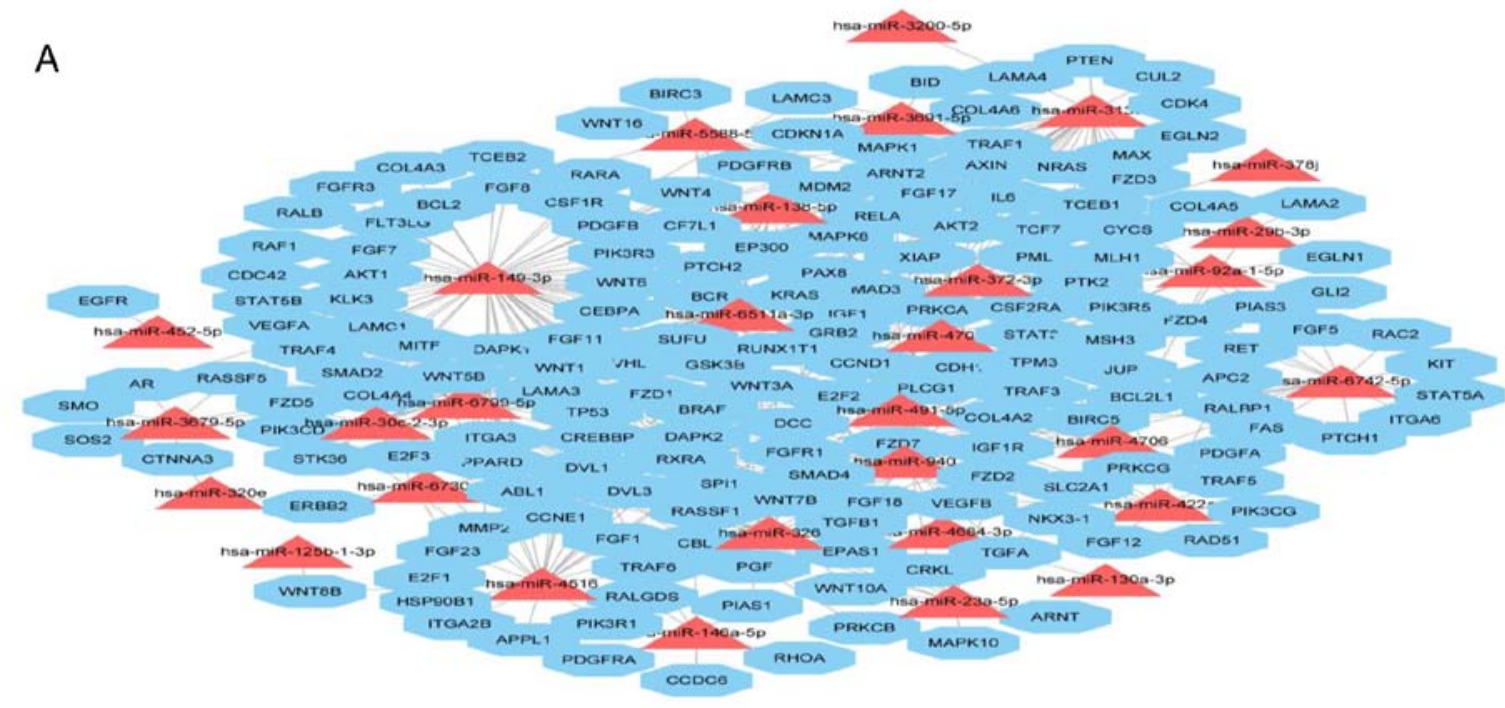

B

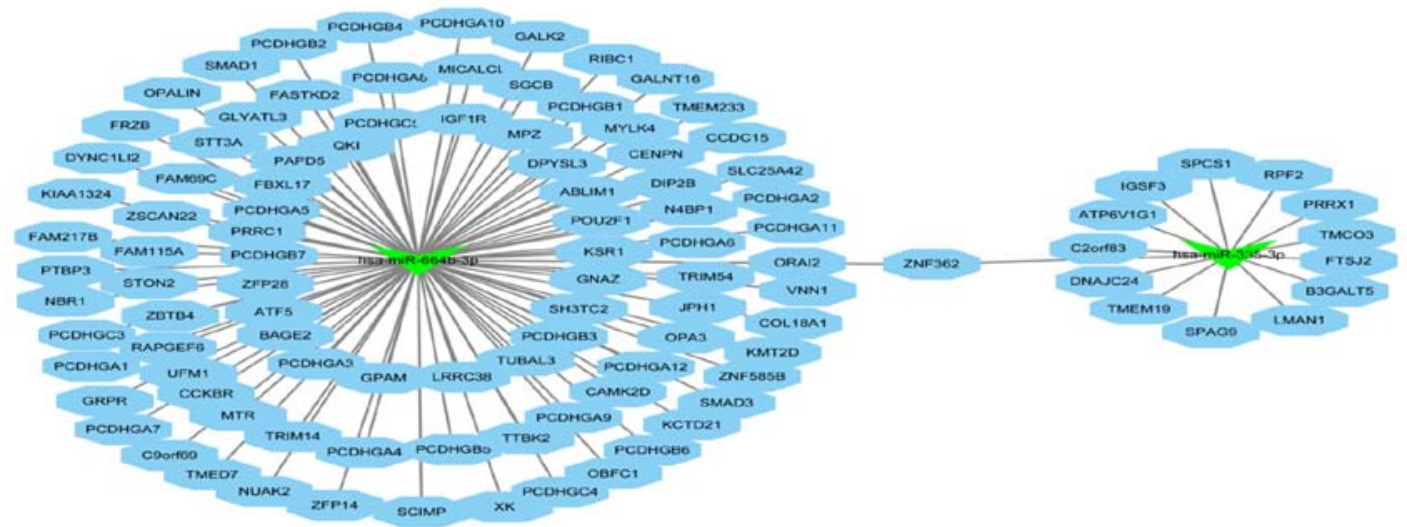

Figure 7. Functional enrichment miRNA-mRNA interaction network analysis of DE miRNA targets. Integrated miRNA/mRNA analysis of potential target mRNAs associated with (A) upregulated or (B) downregulated miRNAs. The triangle refers to DE miRNAs, and the polygon represents the target mRNAs, respectively. Red triangles represent significantly upregulated miRNAs, while green triangles represent significantly downregulated miRNAs. DE, differentially expressed.

A

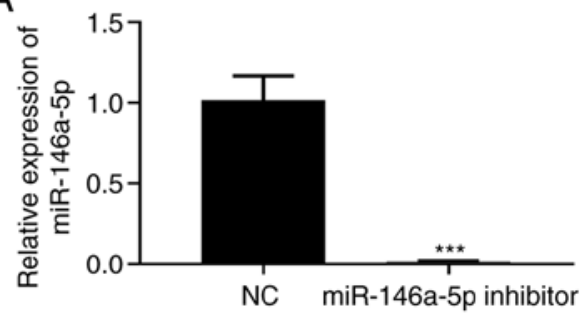

C

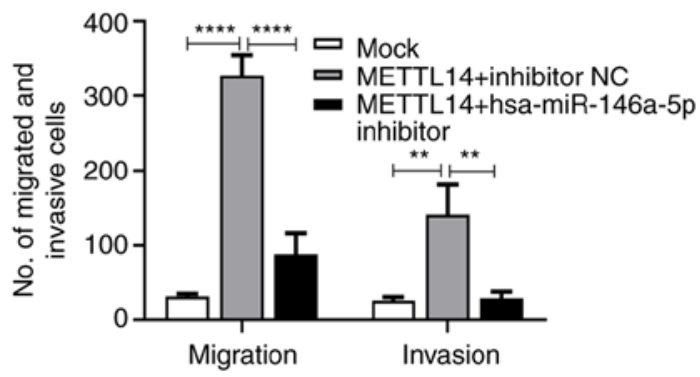

B

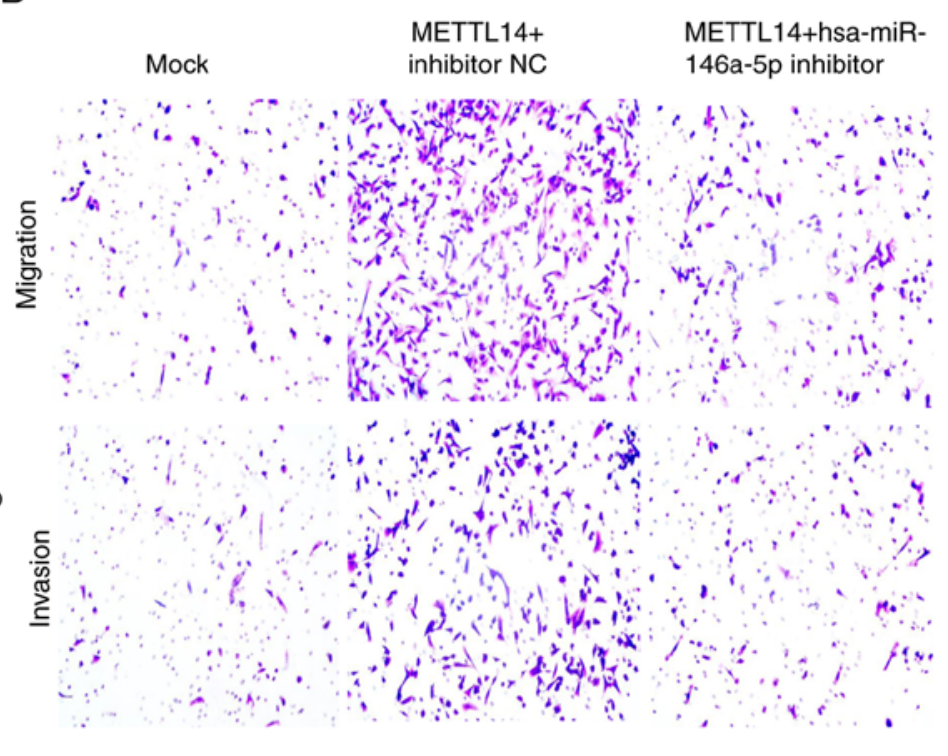

Figure 8. METTL14 promotes migration and invasion of BC cells by regulating hsa-miR-146a-5p. (A) The hsa-miR-146a-5p expression levels were verified by RT-qPCR in MDA-MB-231 cells treated with the hsa-miR-146a-5p inhibitor. (B) The effect of hsa-miR-146a-5p modulated by METTL14 on BC cell migration and invasion. (C) Migration and invasive ability of BC cells were analyzed. Data are the means \pm SDs $(\mathrm{n}=3) .{ }^{* * *} \mathrm{P}<0.01,{ }^{* * * *} \mathrm{P}<0.001$ and ${ }^{* * * * *} \mathrm{P}<0.0001$. METTL14, methyltransferase-like 14; BC, breast cancer. 
hsa-miR-29b-3p, and hsa-miR-3691-5p were present in the network and were expressed as confirmed by RT-qPCR. miR-146a-5p functions as a tumor suppressor in a variety of types of cancer $(38,39)$, its overexpression promotes BC cell proliferation, and it could serve as a new prognostic marker for HER2-positive BC (40). In the present study, miR-146a-5p increased with METTL14 overexpression, which is in accordance with previous reports. Here, overexpression of METTL14 and hsa-miR-146a-5p expression modulated by METTL14 promoted BC cell migration and invasion in vitro. Whether hsa-miR-146a-5p modulated by METTL14 promotes the metastatic potential of BC cells need to be further identified in vivo, which will be the focus of our future research. hsa-miR-146a-5p was enriched in cell adhesion terms through GO functional analysis, suggesting that hsa-miR-146a-5p modulated by METTL14 is associated with metastasis.

In conclusion, abnormal m6A modification in BC was found to be due to the abnormal expression of the m6A methyltransferase METTL14. METTL14 gain- and loss-of-expression was found to regulate m6A levels, and METTL14 overexpression regulated m6A levels to enhance the migration and invasion capacity of MCF-7 and MDA-MB-231 cells. The miRNA/mRNA network was most enriched in the cancer-related pathways, and DE miRNAs were found to be enriched in cell adhesion terms. Moreover, hsa-miR-146a-5p modulated by METTL14 promoted cell migration and invasion. Thus, METTL14 modulates m6A modification and hsa-miR-146a-5p expression, and further promotes cell migration and invasion of breast cancer cells. Taken together, these results indicate that METTL14 may modulate the metastatic potential of breast cancer cells.

\section{Acknowledgements}

Not applicable.

\section{Funding}

This study was supported by the Nanjing Medical Science and Technology Development Project (YKK16093) and the Fundamental Research Funds for the Central Universities (14380389).

\section{Availability of data and materials}

The datasets used and/or analyzed during the present study are available from the corresponding author on reasonable request.

\section{Authors' contributions}

Conceptualization of the study and funding acquisition was achieved by JS. Data collection was carried out by DY, RW and XS. Investigation and analysis of the research data was conducted by DY, RW, XS, LX and YY. Preparation of the original draft was carried out by DY, RW, XS and LX. All authors read and approved the manuscript and agree to be accountable for all aspects of the research in ensuring that the accuracy or integrity of any part of the work are appropriately investigated and resolved.

\section{Ethics approval and consent to participate}

This study was approved by the Human Research Ethics Committee of Nanjing Medical University (Nanjing, Jiangsu, China). All the patients gave written informed consent according to the Declaration of Helsinki, and all experimental methods were conducted following relevant guidelines.

\section{Patient consent for publication}

Not applicable.

\section{Competing interests}

The authors declare that they have no competing interests.

\section{References}

1. Siegel RL, Miller KD and Jemal A: Cancer statistics, 2017. CA Cancer J Clin 67: 7-30, 2017.

2. Nechuta SJ, Caan BJ, Chen WY, Lu W, Chen Z, Kwan ML, Flatt SW, Zheng Y, Zheng W, Pierce JP and Shu XO: Soy food intake after diagnosis of breast cancer and survival: An in-depth analysis of combined evidence from cohort studies of US and Chinese women. Am J Clin Nutr 23: 123-132, 2012.

3. Zhang T, Li J, He Y, Yang F, Hao Y, Jin W, Wu J, Sun Z, Li Y, Chen Y, et al: A small molecule targeting myoferlin exerts promising anti-tumor effects on breast cancer. Nat Commun 9: 3726, 2018.

4. Turner NC, Finn RS, Martin M, Im SA, DeMichele A, Ettl J, Diéras V, Moulder S, Lipatov O, Colleoni M, et al: Clinical considerations of the role of palbociclib in the management of advanced breast cancer patients with and without visceral metastases. Ann Oncol 29: 669-680, 2018

5. Echeverria GV, Powell E, Seth S, Ge Z, Carugo A, Bristow C, Peoples M, Robinson F, Qiu H, Shao J, et al: High-resolution clonal mapping of multi-organ metastasis in triple negative breast cancer. Nat Commun 9: 5079, 2018.

6. Machnicka MA, Milanowska K, Osman Oglou O, Purta E, Kurkowska M, Olchowik A, Januszewski W, Kalinowski S, Dunin-Horkawicz S, Rother KM, et al: MODOMICS: A database of RNA modification pathways-2013 update. Nucleic Acids Res 41 (Database issue): D262-D267, 2013.

7. Frye M, Harada BT, Behm M and He C: RNA modifications modulate gene expression during development. Science 361: 1346-1349, 2018 .

8. Lee M, Kim B and Kim VN: Emerging roles of RNA modification: m(6)A and U-tail. Cell 158: 980-987, 2014.

9. Zhao X, Chen Y, Mao Q, Jiang X, Jiang W, Chen J, Xu W, Zhong L and Sun X: Overexpression of YTHDF1 is associated with poor prognosis in patients with hepatocellular carcinoma. Cancer Biomark 21: 859-868, 2018.

10. Ping XL, Sun BF, Wang L, Xiao W, Yang X, Wang WJ, Adhikari S, Shi Y, Lv Y, Chen YS, et al: Mammalian WTAP is a regulatory subunit of the RNA N6-methyladenosine methyltransferase. Cell Res 24: 177-189, 2014.

11. Wang Y, Li Y, Toth JI, Petroski MD, Zhang Z and Zhao JC: N6-methyladenosine modification destabilizes developmental regulators in embryonic stem cells. Nat Cell Biol 16: 191-198, 2014.

12. Fu Y, Dominissini D, Rechavi G and He C: Gene expression regulation mediated through reversible $\mathrm{m}^{6} \mathrm{~A}$ RNA methylation. Nat Rev Genet 15: 293-306, 2014.

13. Mathiyalagan P, Adamiak M, Mayourian J, Sassi Y, Liang Y, Agarwal N, Jha D, Zhang S, Kohlbrenner E, Chepurko E, et al: FTO-Dependent m6A Regulates Cardiac Function During Remodeling and Repair. Circulation 139: 518-532, 2018.

14. Shen DD, Suo FZ, Song QM, Chang J, Zhang T, Hong JJ, Zheng YC and Liu HM: Development of formaldehyde dehydrogenase-coupled assay and antibody-based assays for ALKBH5 activity evaluation. J Pharm Biomed Anal 162: 9-15, 2019.

15. Panneerdoss S,Eedunuri VK, Yadav $P$, Timilsina S, Rajamanickam $S$, Viswanadhapalli S, Abdelfattah N, Onyeagucha BC, Cui X, Lai Z, et al: Cross-talk among writers, readers, and erasers of $\mathrm{m} 6 \mathrm{~A}$ regulates cancer growth and progression. Sci Adv 4: eaar8263, 2018. 
16. Liu J, Eckert MA, Harada BT, Liu SM, Lu Z, Yu K, Tienda SM, Chryplewicz A, Zhu AC, Yang Y, et al: $\mathrm{m}^{6} \mathrm{~A}$ mRNA methylation regulates AKT activity to promote the proliferation and tumorigenicity of endometrial cancer. Nat Cell Biol 20: 1074-1083, 2018.

17. Lin S, Choe J, Du P, Triboulet R and Gregory R: The m(6)A methyltransferase METTL3 promotes translation in human cancer cells. Mol Cell 62: 335-345, 2016.

18. Zhou S, Bai ZL, Xia D, Zhao ZJ, Zhao R, Wang YY and Zhe H: FTO regulates the chemo-radiotherapy resistance of cervical squamous cell carcinoma (CSCC) by targeting beta-catenin through mRNA demethylation. Mol Carcinog 57: 590-597, 2018.

19. Zhang S, Zhao BS, Zhou A, Lin K, Zheng S, Lu Z, Chen Y, Sulman EP, Xie K, Bögler O, et al: m $^{6}$ A Demethylase ALKBH5 maintains tumorigenicity of glioblastoma stem-like cells by sustaining FOXM1 expression and cell proliferation program. Cancer Cell 31: 591-606.e596, 2017.

20. Cui Q, Shi H, Ye P, Li L, Qu Q, Sun G, Sun G, Lu Z, Huang Y, Yang CG, et al: $\mathrm{m}^{6} \mathrm{~A}$ RNA methylation regulates the self-renewal and tumorigenesis of glioblastoma stem cells. Cell Rep 18 2622-2634, 2017.

21. Hiro-Oki I and Yukihide T: The functions of MicroRNAs: mRNA decay and translational repression. Trends Cell Biol 25 651-665, 2015.

22. Alarcón CR, Lee H, Goodarzi H, Halberg N and Tavazoie SF: N6-methyladenosine marks primary microRNAs for processing. Nature 519: 482-485, 2015.

23. Cai X, Wang X, Cao C, Gao Y, Zhang S, Yang Z, Liu Y, Zhang X, Zhang W and Ye L: HBXIP-elevated methyltransferase METTL3 promotes the progression of breast cancer via inhibiting tumor suppressor let-7g. Cancer Lett 28: 1565, 2017.

24. Ramayo-Caldas Y, Mach N, Esteve-Codina A, Corominas J, Castelló A, Ballester M, Estellé J, Ibáñez-Escriche N, Fernández AI, Pérez-Enciso $M$ and Folch JM: Liver transcriptome profile in pigs with extreme phenotypes of intramuscular fatty acid composition. BMC Genomics 13: 547, 2012.

25. Kozomara A and Griffiths-Jones S: miRBase: Annotating high confidence microRNAs using deep sequencing data. Nucleic Acids Res 42 (Database issue): D68-D73, 2014

26. Wright GW and Simon RM: A random variance model for detection of differential gene expression in small microarray experiments. Bioinformatics 19: 2448-2455, 2003.

27. Agarwal V, Bell GW, Nam JW and Bartel DP: Predicting effective microRNA target sites in mammalian mRNAs. Elife 4: e05005, 2015.

28. Shannon P, Markiel A, Ozier O, Baliga NS, Wang JT, Ramage D, Amin N, Schwikowski B and Ideker T: Cytoscape: A software environment for integrated models of biomolecular interaction networks. Genome Res 13: 2498-2504, 2003.
29. Huang da W, Sherman BT and Lempicki RA: Systematic and integrative analysis of large gene lists using DAVID bioinformatics resources. Nat Protoc 4: 44-57, 2009.

30. Hulsegge I, Kommadath A and Smits MA: Globaltest and GOEAST: Two different approaches for Gene Ontology analysis. BMC Proc 3 (Suppl 4): S10, 2009.

31. Kanehisa M and Goto S: KEGG: Kyoto encyclopedia of genes and genomes. Nucleic Acids Res 28: 27-30, 2000.

32. Livak KJ and Schmittgen TD: Analysis of relative gene expression data using real-time quantitative PCR and the 2(-Delta Delta C(T)) method. Methods 25: 402-408, 2001.

33. Cheng M, Sheng L, Gao Q, Xiong Q, Zhang H, Wu M, Liang Y, ZhuF,Zhang Y,Zhang X, et al: The mA methyltransferase METTL3 promotes bladder cancer progression via $\mathrm{AFF} 4 / \mathrm{NF}-\kappa \mathrm{B} / \mathrm{MYC}$ signaling network. Oncogene 38: 3667-3680, 2019.

34. Sökeland G and Schumacher U: The functional role of integrins during intra- and extravasation within the metastatic cascade. Mol Cancer 18: 12, 2019.

35. Massalha S and Weihs D: Metastatic breast cancer cells adhere strongly on varying stiffness substrates, initially without adjusting their morphology. Biomech Model Mechanobiol 16: 961-970, 2017.

36. Erson-Bensan AE and Begik O: m6A Modification and Implications for microRNAs. Microrna 6: 97-101, 2017.

37. Ma JZ, Yang F, Zhou CC, Liu F, Yuan JH, Wang F, Wang TT, $\mathrm{Xu}$ QG, Zhou WP and Sun SH: METTL14 suppresses the metastatic potential of hepatocellular carcinoma by modulating $\mathrm{N}$-methyladenosine-dependent primary MicroRNA processing. Hepatology 65: 529-543, 2017.

38. Wang C, Zhang W, Zhang L, Chen X, Liu F, Zhang J, Guan S, Sun Y, Chen P and Wang D, et al: miR-146a-5p mediates epithelial-mesenchymal transition of oesophageal squamous cell carcinoma via targeting Notch2. Br J Cancer 118: e12, 2018.

39. Hsieh JY, Huang TS, Cheng SM, Lin WS, Tsai TN, Lee OK and Wang HW: miR-146a-5p circuitry uncouples cell proliferation and migration, but not differentiation, in human mesenchymal stem cells. Nucleic Acids Res 41: 9753-9763, 2013.

40. Gao W, Hua J, Jia Z, Ding J, Han Z, Dong Y, Lin Q and Yao Y: Expression of miR-146a-5p in breast cancer and its role in proliferation of breast cancer cells. Oncol Lett 15: 9884-9888, 2018.

This work is licensed under a Creative Commons Attribution-NonCommercial-NoDerivatives 4.0 International (CC BY-NC-ND 4.0) License. 